\title{
Los Presupuestos de las Corporaciones locales
}

El Título Tercero del Libro Cuarto del texto aprobado por Decreto de 16 de diciembre de 1950 comprende las disposiciones comunes a las Haciendas municipal y provincial, cuyo Capitulo IV se ocupa de los Presupuestos, materia que está desarrollada en treinta artículos, distribuidos en tres Secciones: la primera, para el Presupuesto ordinario; la segunda, para los Presupuestos extraordinarios, y la tercera, para los Presupuestos especiales.

Todo este capitulo es el desarrollo de la Base 65 de la Ley de Bases de 17 de julio de 1945, que se corresponde casi exactamente con el Capitulo III del Título Tercero del Decreto de 25 de enero de 1946, que regulaba provisionalmente las Haciendas locales. En su redacción se advierte, ante todo, un tecnicismo de absoluta fidelidad a la teoría clásica del equilibrio financiero, conservando la esencia de las normas del Estatuto municipal de 1924 y la separación entre Presupuestos ordinarios y extraordinarios, con preceptos de aplicación uniforme para toda clase de. Corporaciones locales.

El régimen económico ordinario de 'a entidad pública se caracteriza, como dice Borght, por la previsión y el empleo regular de los recursos disponibles, para el mantenimiento del equilibrio entre los ingresos y los gastos (1). De aquí la necesidad del presupuesto (budget), cuya aprobación se lleva a cabo por vía legislativa (ley de presupuestos).

(1) Vid. VAn der Borght, Hacienda Pública, tomo I, pág. 27. 
Un país que, en circunstancias normales, quiera obrar prudentemente y que tenga conciencia de la cosa pública, hallará siempre en el presupuesto el instrumento más adecuado para administrarse bien. Pero en los momentos actuales, por circunstancias de todos conocidas, es imposible seguir los sanos principios de la economía de paz. En todos los paises se observa el mismo fenómeno: la carrera desenfrenada deil aumento de los gastos, que obliga a los Gobiernos a imponer una pesada tributación, cuyos peligros han sido denunciados por el economista australiano Dr. Coli Clark, al decir que unos tributos superiores al 25 por 100 de la renta nacional producen presiones inflacionarias muy difíciles de resistir (2).

En el nuevo espíritu de que están infiltradas las tareas del Estado, se viene reconociendo que éste, aparte de sus deberes fundamentales, que consisten en satisfacer de la mejor manera posible las necesidades colectivas ligadas orgánicamente a la existencia y al desarrollo de la Nación, debe adoptar todas las medidas encaminadas al desarrollo económico del país para la utilización productiva de los recursos humanos y materiales, con objeto de garantizar la ocupación total y el incremento de la renta nacional, como igualmente todas las medidas que puedan contribuir, mediante una redistribución social y económicamente justa de la renta nacional, a la mayor productividad de la economía y el mejoramiento del nivel de vida (3).

Cuando los gastos del Estado sobrepasan los ingresos fiscales, el presupuesto es deficitario. El déficit constituye la regla en la política fiscal de hoy en día, sobre todo en una época de

(2) En el estudio o Carta del Banco publicado recientemente por el aNational Eity Bank of New York», se hace ver que, en 1947 y en 1948, los impuestos locaıes y del Estado ascendieron a más del 39 por 100 de la renta nacional en Inglaterra, mientras que en los Estados Unidos la cifra correspondiente fué del 24 por 100. Los ingleses tienen sobre sí unos gravámenes mucho más pesađos que cualquiera de las naciones del mundo occidental. En España, según el Ministro de Hacienda, la carga tributaria que representan los ingresos previstos para 1951, no excede del 15 por 100 de la renta nacional.

(3) Vid. Angelos Angelopulos, Pillie Finance, Holanda; artículo traducido en la revista eImpuesto de la Hacienda Públican, noviembre de 1950, págs. 746 y sigs. 
depresión económica, y de ahí la expresión déficit sistimático. La teoría clásica del presupuesto, tal como ha sido expuesta por Stourm o Jèze, ante los nuevos objetivos de la política fiscal, ha sido reemplazada por la teoría del presupuesto cíclico, propugnado por los economistas suecos, especialmente los Profesores Myrdal y Lindahl, quienes de modo más sistemático han defendido la idea de renunciar al equilibrio como supremo objetivo de la política fiscal, sobre todo en épocas de depresión (4).

Estamos contemplando la transformación y evolución más radical en toda clase de principios. Las mismas teorías de Adolfo Wagner han sido destruídas por Keynes, por motivos puramente económicos ; el Profesor Beveridge, para asegurar la ocupación total, ha trazado otro tipo de presupuesto, basado en una concepción económica del gasto público; el Profesor N. Kaldor sostiene la tesis de un empréstito público continuo para el financiamiento de la ocupación total, etc. (5).

En todo caso se reconoce la necesidad de distinguir entre el presupuesto ordinario y presupuesto extraordinario, denominados también, respectivamente, de impuestos y de empréstitos, de explotación o de consumo y de capital. Myrdal utiliza los términos presupuesto de explotación y presupucsto de capital, desde el punto de vista de la financiación de los gastos, y afirma que el presupuesto ordinario, de impuestos, de consumo o de explotación debe aparecer nivelado. Los gastos ordinarios son los propios del presupuesto ordinario, y para distinguirlos de los extraordinarios, se dice que los primeros son aquellos que se presentan más o menos regularmente cada año, así como

(4) Vid. Mariano Sebastián, Anales de Economía, abril-junio de 1945, páginas 255 y sigs. En nuestras obras Tratado de Presupuestos y Contabilidad Municipal, Presupuestos de las Entidades. Locales y Nuevo Sistema de Contabilidad Municipal se desarrollan con toda amplitud las normas clásicas, que son las aplicables a las Haciendas locales según nuestro Derecho positivo.

(5) Vid. Ramón Ramf́ DE CAP, Efectos económicos del presupuesto equilibrado, en Anales de Economía, enero-marzo de 1950. El autor expone las soluciones propuestas hasta ahora para asegurar una demanda efectiva que absorba el máximo de producción, haciendo un estudio matemático y crítico de la postura de Haavelnio y abogando por él déficit sistemático como método más efecivo para aumentar la renta total. 
los fortuitos de cuantía relativamente insignificante, es decir, que la frontera entre esas dos categorías resülta en cierto grado, imprecisa y convencional (6). Todos los gastos ordinarios deben ser financiados anualmente mediante ingresos reales, mientras que los extraordinarios deben serlo mediante empréstitos. Myrdal supone también que las normas para la limitación de los ingresos han de tener por objeto que, a largo plazo, no solamente los gastos ordinarios, sino también los extraordinarios, han de ser nivelados mediante ingresos reales, lo que significa que el servicio de intereses y amortización se pague con ingresos reales dentro del período que corresponda a la frecuencia con que se repite tales gastos (7).

Pero todos los tipos ideales propuestos para la nivelación presupuestaria, persiguen, a la larga, la sanidad financiera de la Hacienda pública, como es natural. Los gastos totales han de ser suficientes para mantener una ocupación total, dirigiéndose a satisfacer principalmente exigencias sociales, proveyendo a las mismas mediante tributos, mejor que por empréstitos (Beveridge).

Las modernas teorías sobre el presupuesto han surgido ante la imposibilidad de lograr el equilibrio entre gastos e ingresos. Muchas de ellas, pero sobre todo la del presupuesto cíclico, puesta en práctica en Suecia, son ingeniosas y se basan en el presupuesto doble, es decir, de consumo y de capital. En Suecia, el presupuesto de capital está sujeto exclusivamente a consideraciones cíclicas, que permiten una considerable flexibilidad de la política fiscal dentro del ciclo económico. No existe el déficit en el presupuesto ordinario, que queda nivelado al céntimo, merced al ardid que se emplea y que consiste en trasladar a un fondo de nivelación el exceso de los gastos sobre los ingresos. Con objeto de que este fondo mantenga un cierto equilibrio a largo plazo se ha dispuesto que en cada ejercicio se establezca un pro-

(6) Vid. Gunnar Myrdal, Los efectos económicos de la Política Fiscal, traducción por Bengt Becker, 1948, pág. 124.

(7) Modernamente las palabras déficit y superávit han sido reemplazadas por las de subnivelación y supernivelación, respectivamente. 
grama de saneamiento del fondo, en el plazo de diez años. Los déficits son transferidos como partidas negativas al expresado fondo (8).

Pero, sin duda alguna, el verdadero tipo ideal para la nivelación es el que predomina en la Hacienda local, que corresponde a ciertos teoremas normativamente determinados por la economía clásica.

La enumeración de los postulados clásicos del presupuesto puede hacerse de la siguiente forma:

A) Publicidad.-La opinión pública ha de conocer tanto las cifras exactas del presupuesto preventivo como la cuenta general o presupuesto consuntivo.

B) Universalidad.-Es la inscripción en el presupuesto de todos los gastos y de todos los ingresos, cada uno en su lugar, cuyos méritos son indudables. No se debe prescindir en la exposición de los gastos e ingresos de partida alguna, por insignificante que parezca. Este principio encuentra su corolario en la adopción de la regla del presupuesto bruto y lleva implícita la unidad del presupuesto.

C) Unidad de Caja.-Por lo menos contablemente, han de reunirse todos los gastos e ingresos en una sola Caja. A este principio se opone la existencia de Cajas especiales.

D) Especialización.-Aun cuando todos los ingresos se ponen a disposición de la Entidad local, ésta no puede sobrepasar, con ocasión de los distintos gastos, la suma asignada a cada servicio. Además, tanto los gastos como los ingresos han de aparecer convenientemente clasificados.

E) Exactitud.-Tanto los gastos como los ingresos deben evaluarse con la mayor exactitud posible.

F) Nivelación.-El presupuesto debe formarse sin déficit inicial.

Veamos ahora cómo se aplican al presupuesto ordinario es-

(8) Vid. Mariano Sebastín, trabajo citado. En Dinamarca y en Suecia está perfectamente marcada la frontera entre presupuesto de explotación y presupuesto de capital. Myrdal, obra cit., pág. 218. 
tos principios o postulados en el texto artículado de $\mathbf{1 6}$ de diciembre de 1950 :

Publicidad.-El artículo 655 dispone que una vez aprobado el presupuesto, se expondrá al público por quince días hábiles, durante los cuales se admitirán las reclamaciones que se presenten. E1 anuncio de exposición deberá insertarse en el "Boletín Oficial de la Provincian. Los artículos 656 al 660 regulan todo lo relativo a las reclamaciones que deberán ser resueltas por el Delegado de Hacienda. La misma autoridad resuelve sobre el presupuesto. Contra los acuerdos del Delegado de Hacienda en esta materia cabrá recurso ante eil Tribunal Económico-administrativo provincial, cuyo fallo será inapelable. En cuanto al presupuesto consuntivo, el párrafo 2 del artículo 773 prevé la exposición al público, por quince días, de las cuentas de presupuestos, sus justificantes y el dictamen de la Comisión a que se refiere el párrafo 1 del mismo artículo, durante cuyo plazo y ocho días más se admitirán los reparos y observaciones que puedan formularse por escrito.

Universalidad.-Este principio está consagrado en el artículos 649 para los gastos y en el 650 para los ingresos, presidiendo la regla del presupuesto bruto.

Unidad de Caja.-El artículo 740 dispone que todos los fondos deberán ingresar en la Depositaría y ser custodiados en la Caja de la Corporación, de la cual serán claveros el Ordenador de pagos, el Interventor y el Depositario. Se prohibe la existencia de Cajas especiales, no considerándose como tales las cuentas corrientes con Bancos o Sociedades de Crédito debidamente intervenidas.

Especialización.-Preside este principio el artículo 682, a cuyo tenor serán nulos los acuerdos de las Corporaciones y resoluciones de Autoridades municipales y provinciales que habiliten gastos sin crédito suficiente o que creen nuevos servicios sin previa dotación o den mayor extensión a los establecidos, rebasando el crédito correspondiente. Sobre la filiación del estado de gastos, el apartado c) del artículo 649 dispone que se redactará en armonía con el modelo que oficialmente se apruebe, dividiéndolo en capítulos, artículos, conceptos y partidas, nume- 
radas éstas correlativamente en la totalidad del presupuesto; cada concepto contendrá un solo servicio, quedando prohibidas las agrupaciones y el uso de frases que impidan apreciar la naturaleza de los servicios o el coste de cada uno. La enunciación de las exacciones en el estado de ingresos aparecerá en los mismos términos que expresa la Ley, quedando prohibido el empleo de palabras que alteren el verdadero concepto fiscal de aquéllas; y según el apartado $d$ ) del mismo artículo, se redactará en armonía con el modelo que oficialmente se apruebe, dividiéndolo en capítulos, artículos y conceptos, numerados éstos correlativamente en la totalidad del presupuesto.

La regla de la especialización es perfectamente compatible con la concesión de créditos extraordinarios y suplementos de créditos regulados en los artículos 664 y 665 (9).

Exactitud.-El apartado b) del artículo 649 dice que el importe de los créditos será, en los de cuantía fija, igual a la obligación a satisfacer, y en los de carácter variable, se determinará conforme a los proyectos e informe que les sirvan de base, quedando prohibido, en consecuencia, dotar insuficientemente los servicios o rebasar la normal previsión de su coste. Según el apartado c) del artículo 650 los ingresos que en años anteriores hayan dotado un presupuesto, deberán evaluarse en el proyecto del nuevo en cantidad no superior a su rendimiento en el último ejercicio liquidado, a menos que se alteren las tarifas o las condiciones de la recaudación o existan causas excepcionales que justifiquen la previsión de un mayor importe.

Nivelación.-A tenor del artículo 651 ningún presupuesto podrá ser aprobado con déficit, y no podrá elevarse la cuantía cuan-

(9) Ha de acreditarse en el expediente la necesidad y la urgencia de la concesión. Las habilitaciones y suplementos se nutren con el sobrante o superávit de liquidación del último ejercicio, y, en su defecto, por medio de las transferencias de crédito, cuando se den las circunstancias del párrafo 2 del artículo 664 .

El articulo 41 de la Ley de Contabilidad de 1911 prohibe las transferencias, aunque este precepto fué alguna vez dejado en suspenso, como por ejemplo, por Real Decreto del Directorio Militar de 2 de octubre de 1923.

KAREL ENGLis, Introducción a la Ciencia financiera, pág. 285 , considera la transferencia como la operación que se realiza cuando se trasladan los gastos, de manera que lo que se ahorra en una partida se invierte en otra. 
do hubiere resultado déficit en la liquidación anterior, a menos que se justifique plenamente el incremento de ingresos que se calcula.

El verdadero presupuesto es el preventivo, tal como resulta del artículo 648. Al incorporarile las resultas de gastos y de ingresos, como consecuencia de la liquidación del presupuesto del año anterior, en la forma prevista en el artículo 666, se origina el presupuesto refundido, que altera el equilibrio entre gastos e ingresos. Cuando este desnivel significa déficit, el artículo 662 dispone que, en lo posible, se compense con la anulación de gastos voluntarios en la cantidad necesaria, no pudiendo autorizarse ningún gasto de dicha naturaleza en tanto no se adopte tal acuerdo. Si la cantidad rebajable no alcanzase a cubrir el déficit, la diferencia se tendrá en cuenta para cubrirla al formular nuevo presupuesto (10).

El ejercicio económico coincide con el año natural. Las Entidades locales tienen, pues, como el Estado, un período de tiempo durante el cual ejercitan una acción completa administrativa contable (11). Ese período y el ejercicio financiero, que se ha definido también como el tiempo que transcurre desde la apertura hasta el cierre de la contabilidad (12).

Separándose del principio unitario de la Ley de Administración y Contabilidad de la Hacienda pública (13), ya en el Estatuto municipal de 1924 se estableció una franca línea divisoria

(10) Coincidiendo el ejercicio con el año natural, los restos por cobrar y la existencia en Caja en 31 de diciembre, pasan al año siguiente a aresultas de ingrescs , y los restos por pagar en la misma fecha constituyen las aresultas de gastoss. La incorporación de ambas resultas al presupuesto preventivo equilibrado, da origen al presupuesto refundido.

No es el método que se sigue para la confección de los presupuestos generales del Estado, pero la experiencia ha venido demostrando que constituyen un modelo n: uy superior que abraza el conjunto de todas las posibles previsiones.

(11) Vid. Rostagno, Contabilitá d'Stato, 1927, vol. II, pág. 1.

(12) Vid. Cappavianca, Principi fundamentali della Contabilitá dello Stato, 1S66, página 27.

(13) El principio del presupuesto único para el Estado no se hả respetado siempre. Se formaron en 1888-89, 1892-93 y 1896-97. Por Real Decreto-ley de 9 de julio de 1926 se autorizó otro presupuesto extraordinario. También se han confeccionado, con independencia del ordinario desde 1940 durante varios ejercicios, pero en 1947 fué restablecido aquel principio. 
entre presupuestos ordinarios y extraordinarios, prohibiendo severamente el déficit inicial en los primeros; separación que se ha conservado en la Ley de Régimen local, que dedica el Capitulo IV del Libro Tercero a esta materia, con tres Secciones, destinadas: la primera, al presupuesto ordinario; la segunda, a los extraordinarios, y la tercera, a los especiales. El ordinario es de consumo, y el extraordinario, por $\mathbb{l}$ o general, de capital, pues, salvo el caso de calamidades públicas, sólo contendrá gastos de primer establecimiento.

El presupuesto ordinario es el que se forma en cada ejercicio económico (14) para cubrir las obligaciones de carácter permanente o las de carácter temporal que no tengan la naturaleza de gastos de primer establecimiento y para enjugar el déficit de ejercicios anteriores (párrafo 1 del artículo 648). El extraordinario, salvo el caso de calamidades públicas, sólo incluirá gastos de primer establecimiento, prohibiéndose que pueda enjugar el déficit de presupuestos ordinarios (artículo 667), y no pudiendo utilizarse sus dotaciones para fines distintos de los que lo hicieran necesario, a menos que se obtenga previa autorización de la Au-

(14) La historia del año económico de las Entidades locales ha sido la misma de la del Estado, siguiendo análogas vicisitudes. Por Ley de 14 de diciembre último se ha dispuesto que los presupuestos generales del Estado se formen cada dos años. El Ministro de Hacienda hizo ver que la reforma obedece a la necesidad de que precedan a su presentación un mejor estudio y una más amplia experiencia, hablando también de las alteraciones de valores que aconsejan cierta parsimonia ar.tes de proponer modificaciones.

-Si en el pasado el régimen de presupuesto anual, aunque escrito en la ley, no se estimaba en la práctica necesario, en los tiempos actuales creemos que tal sistema ha pasado de innecesario a poco conveniente, puesto que, hay que repetirlo, las variaciones pueden ser más profundas, por tanto, de consecuencias más graves, y ello exige mayor meditación, más larga experiencia, poder medir las consecuencias de una reforma antes de proponerla con apremios de tiempo e incompleto conocimiento de su necesidad o conveniencia.d

- La rápida variación de valores obliga a propuestas de suplementos de crédito, que con dolorosa frecuencia nos vemos obligados a someter a vuestra aprobación. a veces ante apremios de tiempo, a acudir al recurso de decretos-leyes. Pero es más importante y trascendental que una variación de cifras, en definitiva siempre moderada, las variaciones en la estructura del presupuesto, la reforma o reorganización, más o menos profunda, de servicios y las alteraciones importantes del sistema tributario, $y$ en estos aspectos es en lo que estimamos de suma conveniencia dar mayor amplitud para su preparación e implantación si procede.» 
toridad que lo aprobó, cuya decisión será inapelable (art. 676). Finalmente, se consideran especiales los presupuestos de Ensanche y los de Mancomunidades, Agrupaciones y Entidades locales menores (artículo 677).

Como el orden u ordenación es la esencia de toda economía y el sentido de ese orden es equilibrar necesidades y medios, garantizando la cobertura de los gastos, la Ley dedica una gran parte de su articulado a la materia de los recursos, separando los ordinarios de los extraordinarios (estos últimos son objeto del artículo 668), perfilando los de Entidades locales menores (artículo 430), los del presupuesto de Ensanche (artículo 583) y los especiales para amortización de empréstitos municipales (artículos 585 a 594) y provinciales (artículos 630, 631 y 632) que han de incluirse en el presupuesto ordinario. Nada menos que doscientos cuatro artículos, del 529 al 632 , divididos con frecuencia en párrafos y apartados, se ocupan de los ingresos generales, de los que ciento sesenta y seis, del 630 al 594, corresponden a la $\mathrm{Ha}$ cienda municipal, y los treinta y ocho restantes, del 595 al 632, a la Hacienda provincial. La Hacienda estraordinaria halla su expresión, no sólo en el artículo 668 ya citado, sino a lo largo deil capítulo destinado al Crédito local.

También la materia de los gastos ha sido minuciosamente regulada en la Ley, distinguiendo entre ordinarios y extraordinarios (artículo 678) y entre obligatorios y voluntarios (artícu1o 679).

Son gastos ordinarios los que se repiten de una manera regular y constante en cada ejercicio económico aunque experimenten crecimiento, consignados en los Presupuestos ordinarios con carácter obligatorio o voluntario (15).

(15) Con la exacta línea de demarcación que aqui se establece entre gastos ordinarios y extraordinarios, desaparece el lado peligroso de los presupuestos extraordinarios. Las dos palabras están bien definidas en la Ley, y no será posible lievar lo ordinario a lo extraordinario.

La experiencia dirá si los medios que la Ley pone a disposición de las Entidades locales son suficientes, por lo menos, para lograr honestamente la nivelación del presupuesto. A nuestro juicio, en la Ley que comentamos han resultado las Diputaciones provinciales más beneficiadas que los Ayuntamientos. Sobre todo, los 
Son gastos extraordinarios los que independientemente del Presupuesto en que figuren, sean de naturaleza irregular, no periódica, $y$, en particular, los siguientes:

a) los de primer establecimiento relativos a obras y servicios, con absoluta exclusión de todo gasto ordinario de entretenimiento, conservación y explotación, y los demás de naturaleza análoga ;

b) los de calamidades públicas.

Son gastos obligatorios:

a) las deudas exigibles a la Entidad ilocal por cualquier causa: censos, pensiones y cargas; intereses debidos, indemnizaciones, costas y cualesquiera otros de naturaleza análoga;

b) los de prestación de servicios de carácter local encomendados a los Municipios y Provincias por esta Ley, que en relación con las características y medios de cada Entidad local se consideren como mínimo para una elemental gestión que satisfaga las necesidades morales y materiales del vecindario;

c) los de personal y material de todas las oficinas y dependencias de la Entidad local;

d) los de recaudación de recursos legalmente establecidos;

e) los destinados a costear o subvencionar servicios de la Administración general impuestos a los Ayuntamientos y Diputaciones por Ley;

f) los que dimanen del cumplimiento de pactos de Mancomunidad que el Municipio o la Provincia hubieren contratado;

g) los ocasionados por calaminades públicas y los destinados a la ejecución de obras e instalaciones de notoria necesidad y urgencia.

Son gastos voluntarios todos los no comprendidos en los artículos anteriores que, excediendo de las prestaciones mínimas

Municipios rurales continúan en la misma situación descrita en nuestro trabajo Condiciones de vida del Municipio rural, publicado en el aBoletín del Colegio Nacional de Secretarios, Interventores y Depositarios de Administración Localn. La disposición final tercera de la Ley dispone que ésta deberá ser revisada cada cinco años, y el Ministro de la Gobernación, a la vista de los datos y resultados que stiministre la experiencia en la aplicación de sus preceptos, informará al Gobierno y le propondrá, en su caso, las reformas que convenga introducir. 
que les exige esta Ley, pueden realizar discrecional y libremente las Entidades locales con e! fin de mejorar, ampliar y crear servicios y atenciones de su competencia.

Si el presupuesto ordinario coincide con el año natural, el extraordinario tendrá un período de vigencia determinado o indefinido (artículo 667). Si el primero ha de formarse sin déficit inicial (artículo 651), el segundo será siempre nivelado (arțicu1o 667 ).

La tramitación del ordinario se regula en los artículos 653 al 660, distinguiéndose entre el anteproyecto general, que confeccionará el Interventor, y el proyecto, que lo forman el Presidente de la Corporación asistido del Secretario y del Interventor y tomando como base el anteproyecto. La aprobación del proyecto corresponde a la Corporación en pleno, por el voto favorable de la mayoría absoluta del número legal de sus miembros, debiendo estar realizada antes del $\mathbf{1 0}$ de octubre de cada año (artículo 654). Aprobado el presupuesto, se expondrá al público por quince días hábiles, durante los cuales se admitirá reclamaciones (artículo 655), y tales reclamaciones se presentarán al Delegado de Hacienda, por conducto de la Corporación respectiva (artículo 656), otorgándose a los no residentes la facilidad de poder presentarlas en la propia Delegación de Hacienda. Los artículos 656 al 660 regulan todo lo relativo a la personalidad de los reclamantes, casos en que pueden reclamar y plazos para la resolución de reclamaciones y aprobación del presupuesto por 1a primera autoridad económica de la provincia, cuyo acuerdo deberá ser expreso.

Se distingue perfectamente en la Ley el caso de prórroga del presupuesto por un solo año y por un acuerdo consciente de la Corporación (artículo 663), del presupuesto interino (artículo 661), puesto que, si por cualquier causa, al comenzar el ejercicio económico, no estuviese autorizado por el Delegado de Hacienda el presupuesto, regirá interinamente el del ejercicio anterior, con absoluta exclusión de todo gasto voluntario.

La tramitación de los presupuestos extraordinarios es el objeto de los artículos 669 al 674 . El anteproyecto lo forma, bien por su iniciativa o a virtud de acuerdo de la Corporación, el Pre- 
sidente de la misma, asistido del Secretario y del Interventor. Aprobado el proyecto por la Corporación, se expondrá al público durante quince días y se admitirán reclamaciones y observaciones, que serán estudiadas y resueltas en sesión extraordinaria, requiriéndose para aprobar el presupuesto el voto favorable de los dos tercios del número de sus miembros de hecho y, en todo caso, de la máyoría absoluta del número legal. Una vez aprobado, se expone al público con sus anexos por quince dias, durante los cuales se admitirán reclamaciones, que serán cursadas al Ministro o Delegado de Hacienda, según los casos.

En toda la materia de presupuestos se advierte la excepcional importancia de la intervención del Ministerio de Hacienda:

1) Las reclamaciones contra el presupuesto ordinario las resuelve el Delegado de Hacienda en la provincia respectiva, el cual, aunque no haya reclamaciones, decide sobre la aprobación del presupuesto (arts. 658 y 659 ).

2) También el Delegado de Hacienda resuelve sobre los expedientes de modificación de créditos, pero sólo en el caso de presentarse reclamaciones (párrafo 5 del art. 664).

3) Contra las resoluciones del Delegado de Hacienda en materia de presupuestos ordinarios y expedientes de modificación de créditos, cabrá recurso ante el Tribunal Económicoadministrativo provincial, cuyo fallo será inapelable (art. 660 y párrafo 6 del 664).

4) Cuando se trate de presupuestos extraordinarios que no requieran operación de crédito y haya o no reclamaciones, la resolución de éstas y la aprobación o desaprobación de aquéllos corresponde al. Delegado de Hacienda, contra cuyas decisiones sólo se admitirá recurso de alzada ante el Ministro de Hacienda (art. 673).

5) Cuando se trate de presupuestos extraordinarios que requieran operación de crédito, la resolución corresponde al $\mathrm{Mi}$ nistro de Hacienda sin ulterior recurso (art. 674).

6) El Ministro de Hacienda resuelve los expedientes de modificación de créditos de presupuestos extraordinarios dotados con operaciones de crédito (párrafo 2 del art. 675). 
7) Es preciso obtener la previa autorización de la Autoridad que lo aprobó para utilizar las dotaciones de un presupuesto extraordinario en fines distintos de los que lo hicieron necesario (art. 676).

Al presupuesto pueden acompañarse bases de ejecución, y a ellas se refiere el artículo 652, a cuyo tenor tales bases contendrán las disposiciones necesarias para su acertada gestión, estableciendo cuantas prevenciones se consideren precisas o convenientes para la inversión de los gastos y recaudación de los recursos, sin que, en ningún caso, puedan modificar lo legislado para la administración económica, ni comprender preceptos de orden administrativo que requieran legalmente procedimiento y solemnidades distintos del presupuesto. Se comprende que no tengan carácter obligatorio las bases de ejecución, pero sí lo tienen la Memoria explicativa y las certificaciones que se enumeran en el párrafo 2 del artículo 653.

Desde la Real Orden de 6 de abril de $\mathbf{1 9 2 5}$ estaban facultadas las Corporaciones locales para atender con los recursos ordinarios a los gastos de carácter temporal que fuesen de primer establecimiento y que constituyen la materia propia de los presupuestos extraordinarios. Al recoger esta autorización el párrafo 2 del artículo 648 de la Ley se da definitivamente estado legal al principio según el cual es posible que en un solo presupuesto y con recursos exclusivamente ordinarios, sean atendidos toda clase de gastos. Pero se sigue manteniendo la separación de medios para ambos tipos de presupuesto, como hemos visto antes, no pudiendo emplearse ${ }^{-}$los del artículo 668 para atenciones del ordinario, ni para enjugar su déficit. Y claro está que las Entidades locales pueden optar por el presupuesto extraordinario para gastos de primer establecimiento, aun cuando pudiera evitarlo (16).

(16) Las Corporaciones locales que para nivelar sus presupuestos precisen de li.: cupos de compensación que provienen del aFondo de Corporaciones locales» o de! $\alpha$ Fondo de Compensación provincial» no deben hacer uso de la autorización del párrafo 2 del artículo 648 de la Ley, por prohibirlo la Orden del Ministerio de Hacienda de 14 de marzo de 1946. 
Hemos de hacer resaltar la conveniencia del contenido del artículo 675, que permite habilitaciones y suplementos en orden al presupuesto extraordinario, cuando concurran las circunstancias que alli se especifican, cuyos expedientes han de ajustarse al procedimiento establecido para casos análogos del presupuesto ordinario, con la modificación, ya expresada, de quedar conferida la competencia para resolver en todo caso al Ministro de Hacienda cuando estén dotados con operaciones de crédito.

Y finalmente, la Disposición adicional $13 .^{2}$ autoriza a las Corporaciones locales para acordar, antes del 31 de diciembre de 1951, la formación de un presupuesto extraordinario de liquidación de deudas.

Antonio Saura Pacheco 